\title{
Do lateral eye movements increase susceptibility to misinformation? A registered replication
}

\author{
Dustin P. Calvillo ${ }^{1}$ - Ashley S. Emami ${ }^{2}$ \\ Published online: 16 July 2019 \\ (C) The Psychonomic Society, Inc. 2019
}

Keywords False memory $\cdot$ Memory

Eye-movement desensitization and reprocessing (EMDR) has been employed as a treatment for individuals with posttraumatic stress disorder (PTSD; Shapiro, 1989). In EMDR, people perform a series of lateral eye movements while they retrieve memories about a specific event. EMDR has been shown to reduce the vividness and emotionality of traumatic memories (Leer, Engelhard, \& van den Hout, 2014). The efficacy of EMDR, however, has been questioned. Studies have shown that EMDR is less effective for reducing symptoms of PTSD than other treatments are, including cognitive behavioral techniques such as cognitive processing therapy (Cusack et al., 2016) and exposure therapy (Taylor et al., 2003). EMDR has also been criticized for its low incremental utility. That is, there is little to no support for the value it adds to existing treatments for PTSD. Meta-analyses, however, have reported that EMDR is more effective at reducing PTSD symptoms than no treatment (Davidson \& Parker, 2001) and some other psychological treatments (Bisson et al., 2007; Van Etten \& Taylor, 1998).

Components of working memory, including the visuospatial sketchpad (Andrade, Kavanagh, \& Baddeley, 1997) and the central executive (Gunter \& Bodner, 2008), appear to be central to EMDR's effects. EMDR requires dual tasking: Participants simultaneously perform eye movements and retrieve memories. If these two tasks depend on the same pool of resources, eye movements may interfere with the ability to retrieve memories, which degrades the vividness of the

Electronic supplementary material The online version of this article (https://doi.org/10.3758/s13423-019-01641-6) contains supplementary material, which is available to authorized users.

Dustin P. Calvillo

dcalvill@csusm.edu

1 Psychology Department, California State University San Marcos, 333 South Twin Oaks Valley Road, San Marcos, CA 92096, USA

2 Psychology Department, University of Nevada, Las Vegas, NV, USA memory (Maxfield, Melnyk, \& Hayman, 2008). Studies have supported working memory explanations for EMDR's efficacy by demonstrating that other tasks that tax working memory show similar benefits of reducing vividness and emotionality (Engelhard, van den Hout, \& Smeets, 2011; Engelhard, van Uijen, \& van den Hout, 2010; van den Hout et al., 2010). Importantly, eye movements do not seem to reduce vividness and emotionality when memories are not retrieved while performing them (Gunter \& Bodner, 2008). Thus, loading working memory while retrieving memories appears to be a crucial factor in EMDR.

One potential drawback to reducing the vividness of one's memories is that the memories could become more susceptible to distortion. A few studies have explored the effects of eye movements on memory distortion. Christman, Propper, and Dion (2004) examined the effects of eye movements on false recall in the Deese-Roediger-McDermott (DRM) paradigm. They found that lateral eye movements decreased recall of critical lures relative to a stationary eye condition. Parker and Dagnall (2007) found similar results with false recognition in the DRM paradigm. In addition to studies with the DRM paradigm, two studies have examined the effects of eye movements in the misinformation paradigm. Although studies have demonstrated that DRM and misinformation effects poorly correlate across participants and likely arise from different mechanisms (Bernstein, Scoboria, Desjarlais, \& Soucie, 2018; Calvillo \& Parong, 2016; Ost et al., 2013; Patihis, Frenda, \& Loftus, 2018; Zhu, Chen, Loftus, Lin, \& Dong, 2013), these are two common methods to study memory distortion. Parker, Buckley, and Dagnall (2009) examined the effects of eye movements in the misinformation paradigm. Participants viewed a slideshow and heard a verbal commentary of an event. They were then exposed to misleading postevent questions, before performing either lateral eye movements, vertical eye movements, or keeping their eyes stationary. Relative to the other conditions, lateral eye movements increased correct recognition and reduced the false recognition of misinformation. In these experiments, however, 
participants were not asked to retrieve memories while performing eye movements. Working memory explanations of EMDR would not predict a decrease in vividness or emotionality of participants' memories and measures of vividness and emotionality were not reported in these studies.

Houben, Otgaar, Roelofs, and Merckelbach (2018) conducted a study that used an eye movement task that is more similar to those used in EMDR. They had participants watch a video of a car crash, rate the vividness and emotionality of the memory of the video, perform either lateral eye movements or keep their eyes stationary while retrieving memories of the video, rate the vividness and emotionality again, and then read a misleading postevent narrative before taking a recognition test. Houben et al. (2018) found the vividness decreased and emotionality became more positive across the two ratings, but it was not significantly different in the two conditions. Further, participants in the eye-movement condition answered fewer questions correctly and answered more questions with the misinformation alternative than participants in the stationary condition. These results contradict those of Parker et al. (2009), but this contradiction may be explained by methodological differences. Parker et al. had participants perform eye movements after exposure to misinformation, whereas Houben et al. (2018) had them perform eye movements before exposure to misinformation. Houben et al. acknowledged this difference, although Lee, de Jongh, and Hase (2019) claimed that Houben et al. (2018) had ignored the incompatible results of Parker et al. (2009). Houben, Otgaar, Roelofs, and Merckelbach (2019) reiterated the critical differences between Houben et al.'s (2018) method and that of Parker et al. (2009). Additionally, Houben et al. (2018) had participants retrieve their memory of the video while performing eye movements or the control task, whereas Parker et al. (2009) did not.

Houben et al. (2018) demonstrated that a drawback of EMDR was that it could make people more susceptible to misinformation. This has important practical implications. Individuals undergoing EMDR may be exposed to misleading information, and thus their memories of traumatic events may become distorted and, in turn, negatively affect their response to treatment. Furthermore, EMDR has a fairly high association with clients reporting that they had recovered repressed memories in a recent, large-scale survey (Patihis \& Pendergrast, 2018). In legal settings, it may be important that people with PTSD are able to accurately and vividly recollect their memories of the traumatic event. Houben et al.'s (2018) findings are also theoretically relevant. Working memory explanations of EMDR suggest that eye movements should tax working memory and reduce the vividness and emotionality of memories. According to the source monitoring explanation of the misinformation effect (Lindsay \& Johnson, 1989), reduced vividness of an event should make it more susceptible to misinformation. The source monitoring framework claims that the misinformation effect occurs when participants attribute postevent information to the original event. When memory for the original event is vivid, misleading postevent details should be less likely to be misattributed to it.

Because of the practical and theoretical significance of Houben et al.'s (2018) findings and their recommendation that their findings be replicated with a larger sample, we replicated their study. We followed the method of Houben et al. (2018), with two additions. As in Houben et al., participants watched a video, provided vividness and emotionality ratings, performed an eye-movement or control task, provided the vividness and emotionality ratings again, read a misleading postevent narrative, and then took a recognition test for the video. In addition, our recognition test included source memory questions so that we could examine the effects of eye movements on robust false memories (Loftus, 2005). That is, after each recognition test question, participants were asked where they saw the information they used for their responses. If participants stated they remembered seeing an item in the original video when it was only in the postevent narrative, it was considered a robust false memory. Houben et al. (2018) included the Beck Depression Inventory (BDI; Beck \& Steer, 1987) to explore how BDI scores affect vividness and emotionality ratings and if entering BDI scores as a covariate altered the results. We included the BDI-II (Beck, Steer, \& Brown, 1996) and an anxiety symptom measure, the Beck Anxiety Inventory (BAI; Beck, Epstein, Brown, \& Steer, 1988), to explore how depression and anxiety symptoms interact with EMDR.

\section{Method}

\section{Participants}

We conducted a power analysis to determine the sample size of the present experiment. The primary findings of Houben et al. (2018) were that participants who completed the eyemovement exercise were less accurate on the recognition test than control participants $(d=0.88)$, and they accepted more misinformation $(d=0.77)$. These effects were larger than the effect size they used for their power analysis $(d=0.60)$. To be conservative, we used the same effect size that Houben et al. used in their power analysis. Using G*Power (Faul, Erdfelder, Lang, \& Buchner, 2007), we determined that we would need 120 participants ( 60 per condition) to obtain a power of .90 to detect the effect $d=0.60$ with two-tailed $\alpha=.05$. This gave us a power of .99 to detect the smaller effect reported by Houben et al. $(2018 ; d=0.77)$. For our exploratory analyses, we conducted sensitivity analyses and found that this sample size would give us power of .80 to detect correlations of $r=.25$, and to detect main effects and interactions in an ANCOVA with effect sizes of $\eta^{2}=.06$. 
Participants were 120 undergraduate students from California State University San Marcos. There were 34 men and 86 women, and participants ranged in age from 18 to 34 years $(M d n=19.5$ years). They participated in exchange for credit toward the completion of a research requirement in introductory psychology courses.

\section{Design}

The experiment was a one-factor, between-subjects design. Participants were assigned to one of two conditions: eye movement or control. The primary dependent variables were the number of correct and misinformation responses on a recognition test. We also measured source memory to determine the proportion of robust false memories. Additionally, we measured vividness and emotionality of the video, and depression and anxiety scores.

\section{Materials}

Materials and preregistration information are available on the Open Science Framework (https://osf.io/egvx4/).

Misinformation task The materials were the same as those used by Houben et al. (2018), with a few additions. The misinformation task included a video, a postevent narrative, and a recognition test. The video was the same one used by Houben et al., which has also been used in previous trauma film studies (Holmes \& Bourne, 2008). The video depicted an automobile accident that resulted from text messaging while driving. The postevent narrative was also the same one used by Houben et al. (2018). It contained 15 statements, 10 of which were consistent with the video and five of which were inconsistent. The recognition test was also the same as the one used by Houben et al., with an additional component. The recognition test contained 15 two-alternative forced-choice questions, 10 of which had a true and a foil alternative, and five of which had a true and a misinformation alternative. The additional component was the inclusion of a source memory test. After each response to the 15 questions, participants were asked if they knew the response because they (a) saw it in the video only, (b) saw it in the eyewitness statement only, (c) saw it in the video and the eyewitness statement and they were the same, (d) saw it in the video and the eyewitness statement and they were different, or (e) guessed.

Vividness and emotionality ratings Participants rated the vividness of their memory for the video on a visual analog scale from 0 (not at all vivid) to 10 (extremely vivid). They also rated the emotionality of their memory for the video on a scale from 0 (extremely negative) to 10 (extremely positive).
Eye-movement task The eye-movement and control tasks were identical to those used by Houben et al. (2018). Participants were asked to sit $30 \mathrm{~cm}$ away from a computer screen. In the eye-movement condition, participants viewed a gray dot that moved horizontally from left to right in 1-second cycles. This happened for 24 seconds, followed by a $10 \mathrm{sec}-$ ond interstimulus interval, and this sequence repeated for four cycles. For the control condition, participants viewed a stationary gray dot in the center of the screen for 24 seconds, followed by a 10 -second interstimulus interval, and this sequence repeated for four cycles. Both of these tasks were run on E-Prime. All participants were instructed to think about the video of the automobile accident and how they felt while watching the video.

Depressive symptoms Participants' depressive symptoms were assessed with the 21-item BDI-II. Participants selected a response among options for each of the 21 items, and the possible range of scores was from 0 to 63 .

Anxiety symptoms Participants' anxiety symptoms were assessed with the 21-item BAI. Participants selected a response among options for each of the 21 items, and the possible range of scores was from 0 to 63 .

\section{Procedure}

After providing informed consent, participants completed the BDI-II and BAI. Participants were then told that they will see a video of a violent car crash. They were instructed to view the video carefully, as if they were eyewitnesses. After the video, participants completed ratings for vividness and emotionality. Next, participants were assigned to complete either the eyemovement task or the control task. After the eye-movement or control task, participants rated vividness and emotionality of their memory for the video again. Participants then completed a 5-minute filler task. Next, participants read the postevent narrative. After completing another filler task for 5 minutes, participants completed the recognition test. Finally, participants were debriefed. Participants were run individually, and each session took around 30 minutes.

\section{Results}

Our data are available on the Open Science Framework (https://osf.io/egvx4/).

\section{Vividness and emotionality}

We conducted two two-way, mixed-model ANOVAs to test the influence of the eye-movement task on vividness and emotionality ratings. The within-subjects factor was the time 
participants provided the ratings (before or after the eyemovement or control task), and the between-subjects factor was the condition (eye movement or control). The descriptive statistics for the vividness and emotionality ratings are displayed in Table 1. The ANOVA for vividness ratings showed no significant difference between vividness ratings in the two conditions, $F(1,118)=1.52, p=.221, \eta_{\mathrm{p}}{ }^{2}=.01$, a significant effect of time, $F(1,118)=10.08, p=.002, \eta_{\mathrm{p}}{ }^{2}=$ .08 , and a significant interaction between condition and time, $F(1,118)=5.71, p=.018, \eta_{\mathrm{p}}{ }^{2}=.05$. Overall, vividness ratings decreased from the first rating $(M=7.34,95 \%$ CI $[7.07,7.62])$ to the second rating $(M=6.98,95 \%$ CI $[6.68,7.28])$. To examine the interaction, we conducted simple effects tests. The decrease in vividness ratings was significant in the eyemovement group, $t(59)=5.08, p<.001, d=0.66$, but not in the control group, $t(59)=1.40, p=.166, d=0.10$.

The ANOVA for emotionality ratings found no significant difference in ratings among the two conditions, $F(1,118)=$ $0.31, p=.579, \eta_{\mathrm{p}}{ }^{2}=.00$, no significant difference in ratings for the two times, $F(1,118)=3.75, p=.055, \eta_{\mathrm{p}}{ }^{2}=.03$, and no significant interaction, $F(1,118)=0.01, p=.938, \eta_{\mathrm{p}}{ }^{2}=.00$.

\section{Misinformation task}

We calculated three dependent measures from the misinformation task's recognition test. First, we calculated how many correct answers participants provided for the 15 questions. Second, we calculated how many misinformation answers participants provided for the five questions that pertained to the misinformation in the postevent narrative. Finally, we calculated the number of misinformation responses that were accompanied by responses of "a" (saw it in the video only) or "c" (saw it in the video and the eyewitness statement and they were the same) on the source memory test. This served as a measure of robust false memory. Descriptive statistics for these three measures can be found in Table 2 .

We conducted three independent-samples $t$ tests for these dependent measures. There were no differences in how many questions participants correctly answered, $t(118)=0.40, p=.689, d=$ 0.07 , number of misinformation responses, $t(118)=0.10, p=$ $.920, d=0.02$, or number of robust false memories, $t(118)=0.11$, $p=.911, d=0.02$, between participants in the two conditions.

Our exploratory analyses can be found in the Supplementary Material.

\section{Discussion}

Consistent with previous studies (e.g., Leer et al., 2014), we found that the eye-movement exercise decreased self-rated vividness of memories of a traumatic event, whereas a control task did not. The pretest vividness responses from the present study were lower than those of Houben et al.'s (2018) participants, but both studies found decreases from pretest to posttest. Emotion ratings did not significantly decrease after the eye-movement or control task. Collectively, these measures demonstrate that the eye-movement task was effective at reducing the vividness of memory for the video, whereas neither task significantly affected emotion ratings.

The primary purpose of the present experiment was to examine the effects of eye movements on accuracy of memory for the video, the acceptance of misinformation, and on robust false memories. Participants in the eye-movement group did not differ from participants in the control group on any of these three measures. The overall accuracy and number of misinformation items selected were similar to those reported by Houben et al. (2018) — we just did not find the group differences that they did. The finding that the second vividness ratings differed between the two conditions, but the misinformation effect did not suggests that the vividness of memory for an event does not affect susceptibility to misinformation. To further examine the relationship between vividness and misinformation acceptance, we computed the correlation between participants' second vividness rating and their number of misinformation responses. This analysis was not preregistered and was done post hoc. This correlation was significantly positive, $r(118)=.20, p=.027$. We also analyzed this relationship with Houben et al.'s (2018) data and found a nonsignificant correlation, $r(80)=-.04, p=.727$. According to the source monitoring explanation of the misinformation effect (Lindsay \& Johnson, 1989), this relationship should be negative. That is, when participants have more vivid memories of an original event they should be less likely to misattributed postevent information to it. Additional research is needed to understand the relationship between the vividness of memories of an event and susceptibility to misinformation about that event.

We noticed in our exploratory analyses (available in the Supplementary Material) that the depression scores for participants in the present study were considerably higher than those

Table 1 Mean vividness and emotionality ratings before and after participants completed the eye-movement or control activity (EM = eye movement)

\begin{tabular}{llllll}
\hline Condition & \multicolumn{2}{l}{ Vividness } & & \multicolumn{2}{l}{ Emotionality } \\
\cline { 2 - 3 } & Pre [95\% CI] & Post [95\% CI] & & Pre [95\% CI] & Post [95\% CI] \\
\hline EM & $7.32[6.88,7.75]$ & $6.68[6.24,7.11]$ & $2.63[2.13,3.12]$ & $2.82[2.32,3.32]$ \\
Control & $7.37[7.02,7.72]$ & $7.28[6.86,7.70]$ & $2.82[2.30,3.34]$ & $3.00[2.53,3.47]$ \\
\hline
\end{tabular}


Table 2 Mean number of correct responses (out of 15), misinformation responses (out of 5), and robust false memories (out of 5) for participants in each condition (EM = eye movement; 95\% CIs in brackets)

\begin{tabular}{llll}
\hline Condition & Correct responses & Misinformation & Robust false memories \\
\hline EM & $13.17[12.85,13.48]$ & $1.08[0.83,1.33]$ & $0.60[0.38,0.82]$ \\
Control & $13.08[12.81,13.35]$ & $1.10[0.88,1.32]$ & $0.58[0.38,0.79]$ \\
\hline
\end{tabular}

in Houben et al.'s (2018) study. Although Houben et al. used the BDI and we used the BDI-II, the score ranges for these two assessments are the same (0-63). Houben et al. reported a mean BDI score of 4.26 and we found a mean BDI-II score of 10.93. Even though Houben et al.'s and our exploratory analyses did not reveal a vital role of depression scores on misinformation task performance, other studies have demonstrated that depression scores are related to the habitual acceptance of misinformation (Cann \& Katz, 2005). The differences between the two samples may have contributed to the failure to replicate the primary findings. Future studies can continue to explore the relationships between depression symptoms, susceptibility to misinformation, and EMDR.

There were some noteworthy limitations of the present study. To estimate the size of the misinformation effect, studies should have a set of critical items, with participants getting misinformed in half of them and the other half serving as control items, and researchers should counterbalance which items are in the misinformation and control conditions (e.g., Takarangi, Parker, \& Garry, 2006). In the present study, we provided misinformation about five items, and there were no control items. Thus, we cannot estimate the magnitude of the misinformation effect from our results. This is the same procedure, however, that Houben et al. (2018) used. We also used a single video, so the results of this study could be specific to this event. Future studies could examine the effect of EMDR on misinformation with a more diverse set of videos and counterbalanced misinformation and control items. A limitation of our results is that neither eye movements or the control task increased emotion ratings (i.e., made them more positive). The therapeutic benefit of EMDR is that traumatic memories appear less negative in their emotionality. Vividness decreases a few seconds after beginning an eye movement task, whereas emotionality decreases take much longer (Smeets, Dijs, Pervan, Engelhard, \& van den Hout, 2012). Perhaps a longer eye-movement task would have yielded a decrease in emotionality of participants' memory of the event, and this would have resulted in differences in misinformation acceptance between participants in the two conditions. Future research could examine this possibility.

To conclude, we attempted to replicate Houben et al.'s (2018) results that an eye-movement task used in EMDR increases susceptibility to misinformation. We used the same materials and procedure as Houben et al., with two minor additions. We found that the eye-movement task reduced the vividness of the memory, but it did not result in any differences in accurate responding, the acceptance of misinformation, or robust false memories. Although there may be efficacy-based reasons for preferring cognitive-behavioral therapies over EMDR for individuals suffering from PTSD (Cusack et al., 2016), our results suggest that increased susceptibility to misinformation may not be a drawback of EMDR. Additional research is needed to understand the effect EMDR has on the susceptibility to the misinformation effect.

Author note Dustin P. Calvillo, Psychology Department, California State University San Marcos; Ashley S. Emami, Psychology Department, University of Nevada, Las Vegas.

We thank Jonathan Bratton, Madison Keeler, Brianna Reyes, Katie Rodriguez, and Allison Shelton for assistance with data collection. We also thank Sanne Houben for providing the E-Prime script of the eye-movement task.

Open practices statement The data and materials for the experiment in this manuscript are available on the Open Science Framework (https://osf.io/egvx4/), which also contains a copy of the preregistration.

\section{References}

Andrade, J., Kavanagh, D., \& Baddeley, A. (1997). Eye-movements and visual imagery: A working memory approach to the treatment of post-traumatic stress disorder. British Journal of Clinical Psychology, 36, 209-223. doi:https://doi.org/10.1111/j.2044-8260. 1997.tb01408.x

Beck, A. T., Epstein, N., Brown, G., \& Steer, R. A. (1988). An inventory for measuring clinical anxiety: Psychometric properties. Journal of Consulting and Clinical Psychology, 56, 893-897. doi:https://doi. org/10.1037/0022-006X.56.6.893

Beck, A. T., \& Steer, R. A. (1987). Beck depression inventory: Manual. San Antonio, TX: Psychiatric Corporation.

Beck, A. T., Steer, R. A., \& Brown, G. K. (1996). Beck depression inventory-II: Manual. San Antonio, TX: Psychological Corporation.

Bernstein, D. M., Scoboria, A., Desjarlais, L., \& Soucie, K. (2018). "False memory" is a linguistic convenience. Psychology of Consciousness: Theory, Research, and Practice, 5, 161-179. doi: https://doi.org/10.1037/cns0000148

Bisson, J. I., Ehlers, A., Matthews, R., Pilling, S., Richards, D., \& Turner, S. (2007). Psychological treatments for chronic post-traumatic stress disorder: Systematic review and meta-analysis. The British Journal 
of Psychiatry, 190, 97-104. doi:https://doi.org/10.1192/bjp.bp.106. 021402

Calvillo, D. P., \& Parong, J. A. (2016). The misinformation effect is unrelated to the DRM effect with and without a DRM warning. Memory, 24, 324-333. doi:https://doi.org/10.1080/09658211.2015. 1005633

Cann, D. R., \& Katz, A. N. (2005). Habitual acceptance of misinformation: Examination of individual differences and source attributions. Memory \& Cognition, 33, 405-417. doi:https://doi.org/10.3758/ BF03193059

Christman, S. D., Propper, R. E., \& Dion, A. (2004). Increased interhemispheric interaction is associated with decreased false memories in a verbal converging semantic associates paradigm. Brain and Cognition, 56, 313-319. doi:https://doi.org/10.1016/j.bandc.2004. 08.005

Cusack, K., Jonas, D. E., Forneris, C. A., Wines, C., Sonis, J., Middleton, J. C., . . Gaynes, B. N. (2016). Psychological treatments for adults with posttraumatic stress disorder: A systematic review and metaanalysis. Clinical Psychology Review, 43, 128-41. doi:https://doi. org/10.1016/j.cpr.2015.10.003

Davidson, P. R., \& Parker, K. C. (2001). Eye movement desensitization and reprocessing (EMDR): A meta-analysis. Journal of Consulting and Clinical Psychology, 69, 305-316. doi:https://doi.org/10.1037// 0022-006X.69.2.305

Engelhard, I. M., van den Hout, M. A., \& Smeets, M. A. (2011). Taxing working memory reduces vividness and emotional intensity of images about the Queen's Day tragedy. Journal of Behavior Therapy and Experimental Psychiatry, 42, 32-37. doi:https://doi.org/10. 1016/j.jbtep.2010.09.004

Engelhard, I., van Uijen, S., \& van den Hout, M. (2010). The impact of taxing working memory on negative and positive memories. European Journal of Psychotraumatology, 1, 5623. doi:https://doi. org/10.3402/ejpt.v1i0.5623

Faul, F., Erdfelder, E., Lang, A.-G., \& Buchner, A. (2007). G*Power 3: A flexible statistical power analysis program for the social, behavioral, and biomedical sciences. Behavior Research Methods, 39, 175-191. doi:https://doi.org/10.3758/BF03193146

Gunter, R. W., \& Bodner, G. E. (2008). How eye movements affect unpleasant memories: Support for a working-memory account. Behaviour Research and Therapy, 46, 913-931. doi:https://oi. org/10.1016/j.brat.2008.04.006

Holmes, E. A., \& Bourne, C. (2008). Inducing and modulating intrusive emotional memories: A review of the trauma film paradigm. Acta Psychologica, 127, 553-566. doi:https://doi.org/10.1016/j.actpsy. 2007.11.002

Houben, S. T. L., Otgaar, H., Roelofs, J., \& Merckelbach, H. (2018). Lateral eye movements increase false memory rates. Clinical Psychological Science, 6, 610-616. doi:https://doi.org/10.1177/ 216770261875768

Houben, S. T. L., Otgaar, H., Roelofs, J., \& Merckelbach, H. (2019). EMDR and false memories: A response to Lee, de Jongh, and Hase (2019). Clinical Psychological Science, 7, 405-406. doi: https://doi.org/10.1177/2167702619830392

Lee, C. W., de Jongh, A., \& Hase, M. (2019). Lateral eye movements, EMDR, and memory changes: A critical commentary on Houben et al. (2018). Clinical Psychological Science 7, 403-403. doi:https:// doi.org/10.1177/2167702619830395

Leer, A., Engelhard, I. M., \& Van Den Hout, M. A. (2014). How eye movements in EMDR work: Changes in memory vividness and emotionality. Journal of Behavior Therapy and Experimental Psychiatry, 45, 396-401. doi:https://doi.org/10.1016/j.jbtep.2014. 04.004

Lindsay, D. S., \& Johnson, M. K. (1989). The eyewitness suggestibility effect and memory for source. Memory \& Cognition, 17, 349-358. doi:https://doi.org/10.3758/BF03198473
Loftus, E. F. (2005). Searching for the neurobiology of the misinformation effect. Learning \& Memory, 12, 1-2. doi:https://doi.org/10. 1101/lm.90805

Maxfield, L., Melnyk, W. T., \& Hayman, C. G. (2008). A working memory explanation for the effects of eye movements in EMDR. Journal of EMDR Practice and Research, 2, 247-261. doi:https://doi.org/10. 1891/1933-3196.2.4.247

Ost, J., Blank, H., Davies, J., Jones, G., Lambert, K., \& Salmon, K. (2013). False memory $\neq$ false memory: DRM errors are unrelated to the misinformation effect. PLOS ONE, 8(4), e57939.

Parker, A., Buckley, S., \& Dagnall, N. (2009). Reduced misinformation effects following saccadic bilateral eye movements. Brain \& Cognition, 69, 89-97. doi:https://doi.org/10.1016/j.bandc.2008.05. 009

Parker, A., \& Dagnall, N. (2007). Effects of bilateral eye movements on gist based false recognition in the DRM paradigm. Brain \& Cognition, 63, 221-225. doi:https://doi.org/10.1016/j.bandc.2006. 08.005

Patihis, L., Frenda, S. J., \& Loftus, E. F. (2018). False memory tasks do not reliably predict other false memories. Psychology of Consciousness: Theory, Research, and Practice, 5, 140-160. doi: https://doi.org/10.1037/cns0000147

Patihis, L., \& Pendergrast, M. H. (2018). Reports of recovered memories of abuse in therapy in a large age-representative U.S. national sample: Therapy type and decade comparisons. Clinical Psychological Science, 7, 3-21. doi:https://doi.org/10.1177/2167702618773315

Shapiro, F. (1989). Efficacy of the eye movement desensitization procedure in the treatment of traumatic memories. Journal of Traumatic Stress, 2, 199-223. doi:https://doi.org/10.1002/jts.2490020207

Smeets, M. A., Dijs, M. W., Pervan, I., Engelhard, I. M., \& Van den Hout, M. A. (2012). Time-course of eye movement-related decrease in vividness and emotionality of unpleasant autobiographical memories. Memory, 20, 346-357. doi:https://doi.org/10.1080/09658211. 2012.665462

Takarangi, M. K., Parker, S., \& Garry, M. (2006). Modernising the misinformation effect: The development of a new stimulus set. Applied Cognitive Psychology: The Official Journal of the Society for Applied Research in Memory and Cognition, 20, 583-590. doi: https://doi.org/10.1002/acp.1209

Taylor, S., Thordarson, D. S., Maxfield, L., Fedoroff, I. C., Lovell, K., \& Ogrodniczuk, J. (2003). Comparative efficacy, speed, and adverse effects of three PTSD treatments: Exposure therapy, EMDR, and relaxation training. Journal of Consulting and Clinical Psychology, 71, 330-338. doi:https://doi.org/10.1037/0022-006X. 71.2.330

van den Hout, M. A., Engelhard, I. M., Smeets, M. A., Hornsveld, H., Hoogeveen, E., de Heer, E., . . . Rijkeboer, M. (2010). Counting during recall: Taxing of working memory and reduced vividness and emotionality of negative memories. Applied Cognitive Psychology, 24, 303-311. doi:https://doi.org/10.1002/acp.1677

Van Etten, M. L., \& Taylor, S. (1998). Comparative efficacy of treatments for post-traumatic stress disorder: A meta-analysis. Clinical Psychology \& Psychotherapy: An International Journal of Theory and Practice, 5, 126-144. doi:https://doi. org/10.1002/(SICI) 1099-0879(199809)5:3<126::AIDCPP153>3.0.CO;2-H

Zhu, B., Chen, C., Loftus, E. F., Lin, C., \& Dong, Q. (2013). The relationship between DRM and misinformation false memories. Memory \& Cognition, 41, 832-838. doi:https://doi.org/10.3758/ s13421-013-0300-2

Publisher's note Springer Nature remains neutral with regard to jurisdictional claims in published maps and institutional affiliations. 